\title{
Channel Estimation and Tracking for Closed Loop EO-STBC with Differentially Encoding Feedback
}

\author{
Mohamed Nuri Hussin and Stephan Weiss \\ Center for Excellence in Signal \& Image Processing \\ Department of EEE, University of Strathclyde ,Glasgow, Scotland, UK \\ \{mohamed.hussin,stephan.weiss\}@eee.strath.ac.uk
}

\begin{abstract}
Extended orthogonal space time block coding (EOSTBC) can achieve high transmit diversity over a multipleinput multiple-output (MIMO) channel. To do so, it requires channel state information on the transmitter side, which needs to be estimated and fed back from the receiver. Therefore, this paper explores an estimation and tracking scheme by means of a Kalman filter, which is integrated with EO-STBC detection and exploits the smooth evolution of the channel coefficients by applying differential feedback. For slow fading, we propose the inclusion of a drift vector in the Kalman model, which is motivated by a second order approximation of the underlying channel model and can be shown to offer advantages in terms of temporal smoothness when addressing channels whose coefficient trajectories evolve smoothly.
\end{abstract}

\section{INTRODUCTION}

Amongst various space-time block coding (STBC) schemes [1], [2], orthogonal STBC (O-STBC) has low decoding complexity and can achieve full diversity gain at a code rate equal to one. However, for complex constellation and more than two transmit antennas, the code rate must be relaxed in order to admit full diversity. In contrast, quasi-orthogonal STBC (QO-STBC) using four transmit antennas achieves full code rate at the expense of a loss in diversity gain [3]. Constellation rotation was proposed for QO-STBC in order to mitigate symbols interference to attain full code rate and full diversity [4] at the cost of an increase in system complexity.

Extended O-STBC (EO-STBC) inherits O-STBC's low decoding complexity [5], but at a code rate of one, and full diversity gain plus an additionally array gain. The latter is realised by appropriate beamsteering, which depends on the channel estimate and needs to be fed back in a closed-loop architecture from the receiver to the transmitter.

The EO-STBC scheme in [6] has investigated quantised feedback of optimum parameters. When operated in a fading scenario, the channel coeff cients are slowly time-varying due to small Doppler spread and large coherence times [7]. This smooth evolution of channel coeff cients has motivated the investigation of differential feedback in [8], which shows that improved performance to standard quantisation is possible at half the feedback bandwidth over a range of realistic Doppler spreads.

EO-STBC requires accurate knowledge of channel state information (CSI) both at the transmitter for beamsteering, as well as at the receiver for decoding [9]. Channel estimation is therefore crucial, and can only be avoided through the use of STBC based on differential or double differential modulation [10], [11]. This alleviated the need for CSI, but requires the channel to remain stationary for a few successive symbol periods and incurs a performance degradation by $3 \mathrm{~dB}$ and $6 \mathrm{~dB}$, respectively, compared to coherent modulation [10], [11].

In order to obtain CSI, joint channel tracking and symbol detection schemes have been proposed, such as the decisiondirected (DD) approach in [12] for O-STBC. In order to link the decoding stage with the tracking stage, a Kalman $\mathrm{f}$ lter was adopted in [12], which can be effectively based on an autoregressive (AR) state-space model of order $p$ (AR- $p$ ) for the time-varying channel. For example, an AR-1 model has been used in [13]. In order to allow a better prediction, particularly for slow-fading scenarios and in light of the fact that for STBC the channel has to be predicted ahead over two symbol periods, an AR-2 model has recently been proposed in [14].

In this paper, we extend the work in [8] by proposing a channel estimation and tracking scheme based on an AR-2 model-based Kalman flter. This system needs to be adapted in order to interlink with the EO-STBC symbol detection in the Kalman flter's correction step. Therefore, Sec. II provides a brief overview over EO-STBC with differential feedback. In Sec. III, channel estimation and tracking by means of a Kalman flter is introduced. Results are presented in Sec. IV, and Sec. V provides a summary and conclusions.

In our notation, lower and upper-case case bold face variables such as $\mathbf{h}$ and $\mathbf{H}$ represent vector and matrix quantities, respectively. For a matrix $\mathbf{H}$, the transpose is denoted by $\mathbf{H}^{\mathrm{T}}$, the Hermitian by $\mathbf{H}^{\mathrm{H}}$, and the complex conjugate by $\mathbf{H}^{*}$. The statistical expectation operator is given by $\mathcal{E}\{\cdot\}$.

\section{SYSTEM MODEL}

\section{A. Closed Loop EO-STBC}

In this section we consider a model for a time-varying multiple input single output (MISO) system. Based on EOSTBC transmission as outlined in Fig. 1, the forward link has four transmit antennas and a single receive antenna. The feedback link is required to return information on the steering angles $\vartheta_{1}$ and $\vartheta_{2}$, with which the frst and third antenna signal are modif ed. We will later see that by judiciously selecting these angles based on knowledge of the channel at the receiver, the combined diversity and array gain of the 


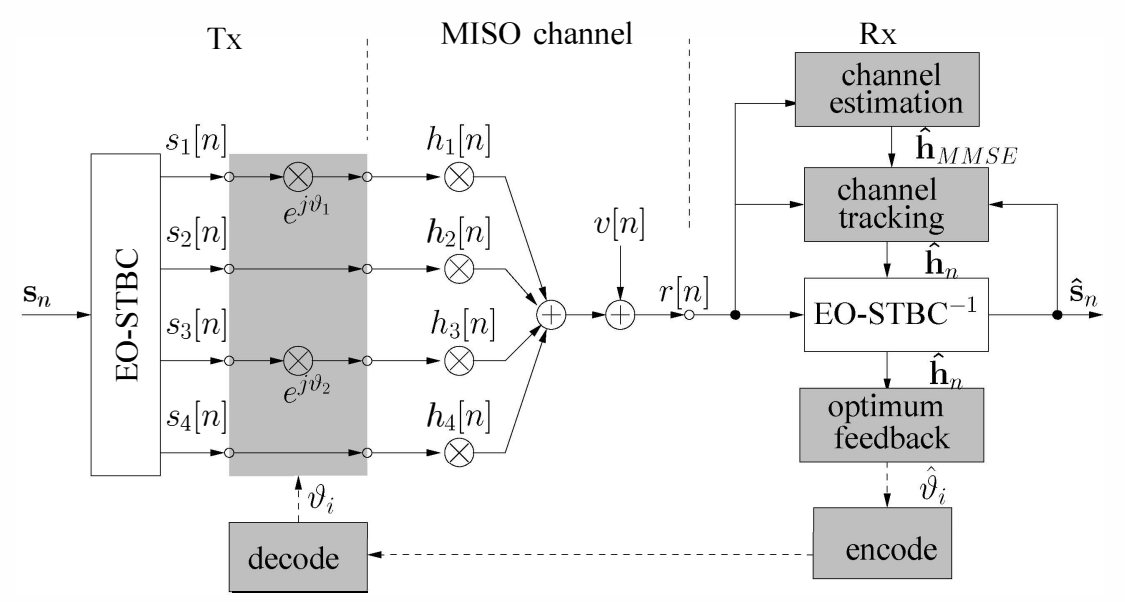

Figure 1. EO-STBC system with channel estimation and tracking in the receiver to aid symbol detection and estimation of the optimum beamsteering angles $\vartheta_{1}$ and $\vartheta_{2}$, which are differentially encoded and returned to the transmitter.

system can be maximised. For simplicity, we assume below that the feedback channel is error free with no latency. Below, the system components in Fig. 1 are reviewed.

1) Transmit Signal and Channel: The channel is timevarying with a normalised angular Doppler frequency $\Omega_{D}=$ $\omega_{D} T_{\mathrm{s}}$, with $T_{\mathrm{s}}$ being the symbol rate. Due to mobility and multipath propagation, the carrier frequency will experience Doppler spread. For slow fading channels, each channel coef$\mathrm{f}$ cient evolves smoothly over time. The received signal $r[n]$ is characterised by

$$
r[n]=\mathbf{h}_{n}^{T} \boldsymbol{\Lambda}_{n} \mathbf{s}_{n}+v[n],
$$

where $\mathbf{s}_{n}$ is the transmit data vector, $\boldsymbol{\Lambda}_{n}$ is a unitary diagonal matrix, and $v[n]$ is zero mean uncorrelated complex Gaussian noise with variance $\sigma_{v}^{2}$. The vector $\mathbf{h}_{n} \in \mathbb{C}^{4}$,

$$
\mathbf{h}_{n}=\left[\begin{array}{lll}
h_{1, n} & \ldots & h_{4, n}
\end{array}\right]^{T}
$$

contains spatially independent and identically distributed widesense stationary (WSS) Rayleigh fading channel coeff cients. The spatio-temporal covariance matrix of these coeff cients is therefore given by

$$
\mathbf{R}_{h}[\tau]=\mathcal{E}\left\{\mathbf{h}_{n-\tau} \mathbf{h}_{n}^{H}\right\}=4 \sigma_{h}^{2} J_{0}\left(\Omega_{D} \tau\right) \mathbf{I} \quad,
$$

where $J_{0}(\cdot)$ is the zeroth order Bessel function of $\mathrm{f} \mathrm{rst} \mathrm{kind} \mathrm{[7],}$ and $\sigma_{h}^{2}$ the variance of the Rayleigh distributed channel coeff cients. With EO-STBC encoding over two successive time slots, the selection of the transmit vector is given by

$\mathbf{s}_{n}=\left\{\begin{array}{lll}\frac{1}{2}[s[n], s[n], s[n+1], s[n+1]]^{T}, & n & \text { even } \\ \frac{1}{2}\left[-s^{*}[n],-s^{*}[n], s^{*}[n-1], s^{*}[n-1]\right]^{T} & n & \text { odd. }\end{array}\right.$

The diagonal beamsteering matrix $\boldsymbol{\Lambda}_{n}$

$$
\boldsymbol{\Lambda}_{n}=\operatorname{diag}\left\{e^{j \hat{\vartheta}_{1}[n]}, 1, e^{j \hat{\vartheta}_{2}[n]}, 1\right\}
$$

effectively rotates the channel taps $h_{1}[n]$ and $h_{3}[n]$. Since $\boldsymbol{\Lambda}_{n}$ is unitary, it has no effect on the transmitted power.

The feedback angles $\hat{\vartheta}_{1}[n]$ and $\hat{\vartheta}_{2}[n]$ can be absorbed into the estimated channel. Let the estimated channel with incorporated angles can be given by

$$
\overline{\mathbf{h}}_{n}=\boldsymbol{\Lambda}_{n} \mathbf{h}_{n}
$$

where $\mathbf{h}_{n}$ is the true transmission channel. At the receiver side, the parameters $\hat{\vartheta}_{1}$ and $\hat{\vartheta}_{2}$ are extracted from the estimated channel coeff cients. Therefore a simple correction

$$
\mathbf{h}_{n}=\boldsymbol{\Lambda}_{n}^{-1} \overline{\mathbf{h}}_{n}=\boldsymbol{\Lambda}_{n}^{*} \overline{\mathbf{h}}_{n} .
$$

can extract the true channel coeff cients from the estimate incorporating the known beamsteering angles.

2) Received Signal: As in a standard STBC system, the receiver gathers two subsequent samples in a vector $\mathbf{r}_{n}$ such that

$$
\mathbf{r}_{n}=\left[\begin{array}{c}
r[n] \\
r^{*}[n+1]
\end{array}\right]=\mathbf{H}_{n} \mathbf{s}_{n}+\mathbf{v}_{n},
$$

based on the equivalent transmit vector $\mathbf{s}_{n}=[s[n], s[n+1]]^{T}$, equivalent noise vector $\mathbf{v}_{n}=\left[v[n], v^{*}[n+1]\right]^{T}$, and the spacetime equivalent transmission channel matrix $\mathbf{H}_{n}$. The latter can be formulated as

$$
\mathbf{H}_{n}=\left[\begin{array}{ll}
h_{11}[n] & h_{12}[n] \\
h_{21}[n+1] & h_{22}[n+1]
\end{array}\right] . .
$$

The components of $\mathbf{H}_{n}$ are a mixture of channel coeff cients and rotations due to beamsteering with

$$
\begin{aligned}
h_{11}[n] & =e^{j \vartheta_{1}[n]} h_{1}[n]+h_{2}[n] \\
h_{12}[n] & =e^{j \vartheta_{2}[n]} h_{3}[n]+h_{4}[n] \\
h_{21}[n+1] & =e^{-j \vartheta_{2}[n+1]} h_{3}^{*}[n+1]+h_{4}^{*}[n+1] \\
h_{22}[n+1] & =-e^{-j \vartheta_{1}[n+1]} h_{1}^{*}[n+1]-h_{2}^{*}[n+1] .
\end{aligned}
$$

3) Signal Detection: Detection is performed over a block duration consisting of two successive symbols periods. The decision statistic vector $\hat{\mathbf{s}}_{n}=\left[\hat{s}[n], \hat{s}^{*}[n+1]\right]^{T}$ can be obtained via linear combination with space-time equivalent estimated channel matrix $\hat{\mathbf{H}}_{n}$ as

$$
\hat{\mathbf{s}}_{n}=\hat{\mathbf{H}}_{n}^{H} \mathbf{H}_{n} \mathbf{s}_{n}+\tilde{\mathbf{v}}_{n} .
$$

where $\tilde{\mathbf{v}}_{n}=\hat{\mathbf{H}}_{n}^{H} \mathbf{v}_{n}$ is the noise after decoding. Let the matrix $\mathbf{G}_{n}=\mathbf{H}^{H}{ }_{n} \mathbf{H}_{n}$, which can be decomposed into 
$\mathbf{G}_{n}=\mathbf{H}^{H}{ }_{n} \mathbf{H}_{n}+\left(\Delta \mathbf{H}_{n}\right)^{H} \mathbf{H}_{n}$. The matrix $\Delta \mathbf{H}_{n}$ represents the error due to both estimation and time variation, which creates inter-symbol interference in the detection process and increases the noise foor. In the absence of estimation errors, i.e. $\hat{\mathbf{H}}_{n}=\mathbf{H}_{n}$, any errors are only due to time-variations and the elements of $\mathbf{G}_{n}$ become

$$
\mathbf{G}_{n}=\left[\begin{array}{ll}
g_{11}[n] & g_{12}[n] \\
g_{21}[n] & g_{22}[n]
\end{array}\right],
$$

with

$$
\begin{aligned}
g_{11}[n]= & \sum_{m=1}^{2}\left|h_{m}[n]\right|^{2}+\sum_{m=3}^{4}\left|h_{m}[n+1]\right|^{2} \\
& +\Re\left\{e^{j \vartheta_{1}[n]} h_{1}[n] h_{2}^{*}[n]\right. \\
& \left.+e^{j \vartheta_{2}[n+1]} h_{3}[n+1] h_{4}^{*}[n+1]\right\} \\
g_{12}[n]= & e^{j\left(\vartheta_{2}[n]-\vartheta_{1}[n]\right)} h_{1}^{*}[n] h_{3}[n] \\
& -e^{j\left(\vartheta_{2}[n+1]-\vartheta_{1}[n+1]\right)} h_{1}^{*}[n+1] h_{3}[n+1] \\
& +e^{-j \vartheta_{1}[n]} h_{1}^{*}[n] h_{4}[n] \\
& -e^{-j \vartheta_{1}[n+1]} h_{1}^{*}[n+1] h_{4}[n+1] \\
& +e^{j \vartheta_{2}[n]} h_{2}^{*}[n] h_{3}[n] \\
& -e^{j \vartheta_{2}[n+1]} h_{2}^{*}[n+1] h_{3}[n+1] \\
& +h_{2}^{*}[n] h_{4}[n]-h_{2}^{*}[n+1] h_{4}[n+1] \\
g_{21}[n]= & g_{12}^{*}[n] \\
g_{22}[n]= & \sum_{m=1}^{2}\left|h_{m}[n+1]\right|^{2}+\sum_{m=3}^{4}\left|h_{m}[n]\right|^{2} \\
& +\Re\left\{e^{j \vartheta_{1}[n+1]} h_{1}[n+1] h_{2}^{*}[n+1]\right.
\end{aligned}
$$

The maximisation of the received gain is coupled to the maximisation of the on-diagonal elements of $\mathbf{G}_{n}$, which is achieved by setting

$$
\begin{aligned}
\vartheta_{1}[n] & =-\angle\left\{h_{1}[n] h_{2}^{*}[n]\right\} \\
\vartheta_{1}[n+1] & =-\angle\left\{h_{1}[n+1] h_{2}^{*}[n+1]\right\} \\
\vartheta_{2}[n] & =-\angle\left\{h_{3}[n] h_{4}^{*}[n]\right\} \\
\vartheta_{2}[n+1] & =-\angle\left\{h_{3}[n+1] h_{4}^{*}[n+1]\right\} .
\end{aligned}
$$

However, unlike in the stationary case [6], the off-diagonal elements $g_{12}[n]$ and $g_{21}[n]$ are now f nite and create intersymbol interference in the process of detecting $s[n]$ and $s[n+$ $1]$.

\section{B. Differential Quantised Feedback}

For many wireless applications, the maximum Doppler shift $\Omega_{D}$ can be considered small. This leads to a smooth evolution of channel coeff cients, which enables differentially encoding single bit feedback over a realistic range of maximum Doppler shifts $\Omega_{D}$ [8]. Differentially quantised feedback requires optimisation of the quantiser step size $\mu$, which causes a trade-off between slope overload due to too slow tracking for small values of $\mu$, and too coarse quantisation for larger values of $\mu$ that causes poor performance irrespective of the rate of change. Below, we will aim to construct channel estimation and tracking that can work together with the differential feedback previously explored in [8].

\section{Channel Estimation And Tracking}

The EO-STBC receiver requires the knowledge of the channel coeff cients both for detecting symbols as well as for computing the optimum phase angles $\vartheta_{i, n}, i=\{1,2\}$, to be used for beamsteering. Therefore, below an approach to track the channel coeff cients is presented, which is initialised and interleaved with channel estimation steps.

\section{A. Channel Estimation}

An initial channel estimate for the MISO system $\mathbf{h}_{n}$ can be obtained by transmitting a training sequence over $L$ symbols. If this transmit data is assembled in a data matrix $\mathbf{S}_{n} \in \mathbb{C}^{L \times 4}$ and transmitted without beamsteering, the minimum mean square error (MMSE) solution for $\hat{\mathbf{h}}_{n}$ for $L \geq 4$ can be calculated as

$$
\hat{\mathbf{h}}_{\mathrm{MMSE}}=\left(\mathbf{S}^{H}{ }_{n} \mathbf{S}_{n}+\gamma_{n}^{-1} \mathbf{I}\right)^{-1} \mathbf{S}^{H}{ }_{n} \mathbf{r}_{n},
$$

where $\mathbf{r}_{n} \in \mathbb{C}^{L}$ is the vector of received samples, and $\gamma_{n}$ is the SNR at the receiver. The formulation in (23) includes the ZF estimate with the case $\gamma_{n} \rightarrow \infty$.

For $L=2, \mathbf{S}^{H}{ }_{n}=\left[\mathbf{s}_{n} \mathbf{s}_{n+1}\right] \in \mathbb{C}^{2 \times 4}$ with $\mathbf{s}_{n}$ def ned in (3) is the orthogonal data matrix. The MMSE solution requires the left pseudo-inverse [15], and due to orthogonality of $\mathbf{S}_{n}$ simplif es to

$$
\hat{\mathbf{h}}_{\mathrm{MMSE}}=\frac{\gamma_{n}}{1+\gamma_{n}} \mathbf{S}^{H}{ }_{n} \tilde{\mathbf{r}}_{n}
$$

Either (23) or (24) can be used to initialise the subsequent Kalman tracking, and can also be employed to re-initialise the tracking system in periodic intervals.

\section{B. Kalman-Based Tracking \& Detection}

The Kalman flter approach has been utilised widely for channel tracking. Here, we select a simple AR-2 model which contains a so called drift vector $\Delta \mathbf{h}_{n}$ in order to better exploit the smooth evolution of the channel coeff cients as compared to the standard AR-1 model utilised in most publications. An accurate system can be built from a prediction model exploiting the temporal correlation expressed in (2) [7], while here a simplif ed heuristic state-space model

$$
\left[\begin{array}{c}
\mathbf{h}_{n} \\
\boldsymbol{\Delta} \mathbf{h}_{n}
\end{array}\right]=\left[\begin{array}{ll}
\mathbf{I}_{n} & \mathbf{I}_{n} \\
\mathbf{0}_{n} & \mathbf{I}_{n}
\end{array}\right] \cdot\left[\begin{array}{c}
\mathbf{h}_{n-1} \\
\Delta \mathbf{h}_{n-1}
\end{array}\right]+\left[\begin{array}{c}
\underline{0} \\
\mathbf{w}_{n}
\end{array}\right]
$$

is invoked, containing $\mathbf{w}_{n}$ as the drift error with variance $\sigma_{w}^{2}$, is the only parameter which can be tuned to control the drift of $\Delta \mathbf{h}_{n}$.

This state-space model is incorporated in the Kalman flter algorithm outlined in Tab. I, where an a-priori estimate obtained in a prediction step is improved based on the detected symbols contained in a data vector $\mathbf{s}_{n}$, yielding the a-posteriori estimate $\mathbf{h}_{n \mid n}$ of the channel as in Fig. 1.

\section{Decision-Directed Channel Tracking}

The Kalman flter algorithm in Tab. I is updated at every symbol period based on the detected data. In this section, we want to customise the Kalman flter algorithm to take the EOSTBC setup as depicted in Fig.1 into account. While Tab. I 
Table I

KALMAN FILTER TO ITERATIVELY ESTIMATE AND TRACK $\hat{\mathbf{h}}_{n \mid n}$.

already assumes that $\mathbf{s}_{n}$ has been suitably detected, below we formalise the decision directed approach in [12] for the purpose of our proposed architecture. Thereby, two a-priori estimates are performed to predict the time-varying channel vectors during the current EO-STBC block. Thereafter, both a-priori estimates are updated according to the algorithm in Tab. II.

\section{Impact of Beamsteering on Channel Estimation}

According to Fig.1, the feedback angles $\vartheta_{1}[n]$ and $\vartheta_{2}[n]$ are absorbed into the original channels of antennas one and three. Fig 2 shows the channel variations when $\Omega_{D}=0.003 \pi$. Fig. 2(a)-(d) show real and imaginary parts of the trajectory of the true channel coeff cients $h_{i}[n], i=\{1,2\}$ and, in case of $h_{1}[n]$, real and imaginary part of the trajectory of the modif ed coeff cient (dashed curves) in Fig. 2(a)-(b).

The trajectory of the feedback angle $\vartheta_{1}[n]$ is shown in Fig. 2(e). In order to avoid divergence of the Kalman flter, a re-initialisation with an MMSE estimate driven by pilot symbols is performed every $K$ symbols, with the default value set to $K=12$. This is necessary, since e.g. a coeff cient trajectory passing close to the origin leads to rapidly changing angles, and therefore a higher degree of non-stationarity. Particularly Fig. 2(a)-(b) very clearly show how beamsteering angles affect the rate of change in terms of the coeff cients.

Table II

KALMAN-BASED CHANNEL OPERATION WITH DECISION-DIRECTED UPDATING OVER TWO SUCCESSIVE SYMBOL PERIODS.

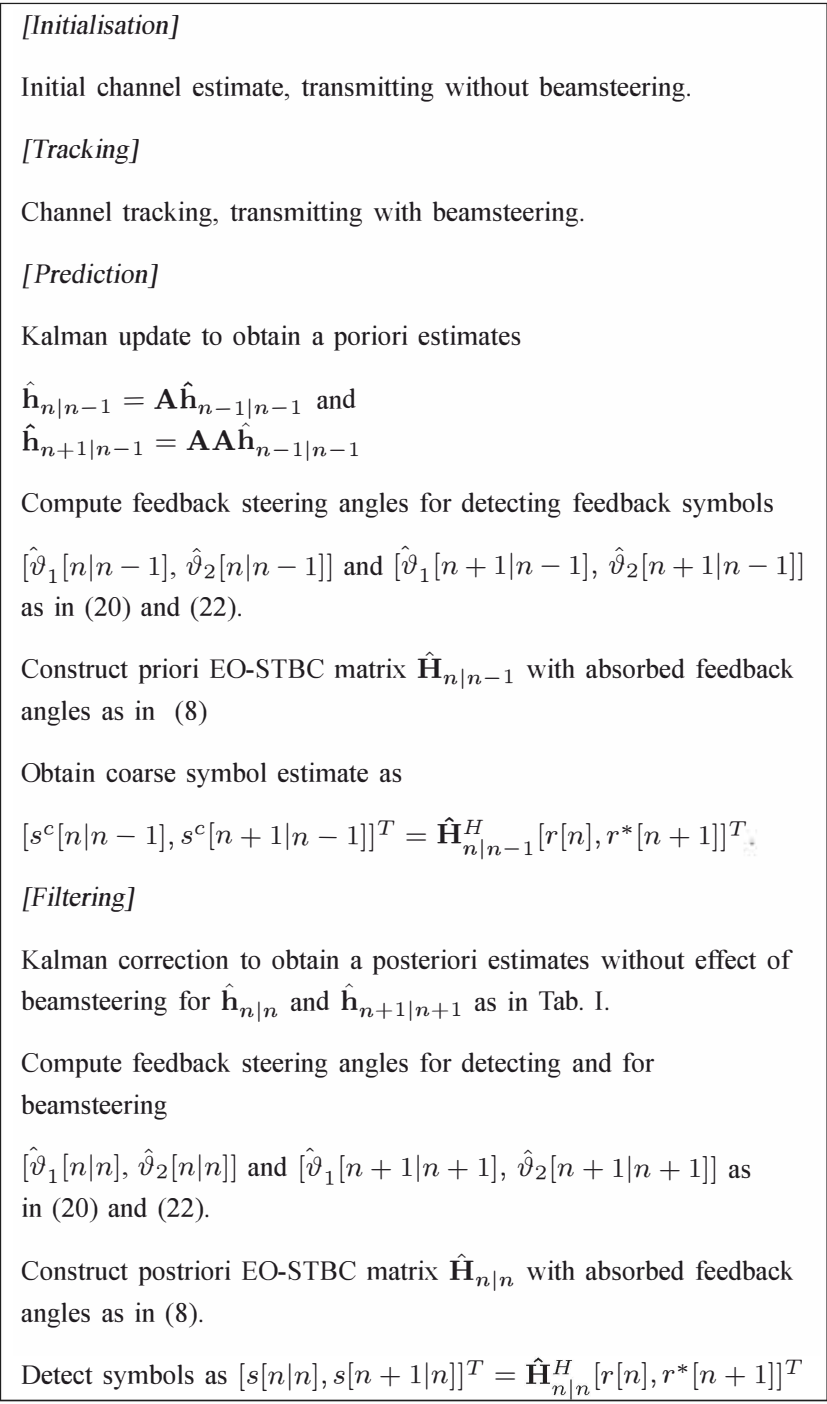

Although the problem occurs in channel $h_{1}[n]$ due to the beamsteering, the impact is felt across all channel coeff cients.

\section{Simulations AND Results}

In the following, the BER performance of EO-STBC with differential feedback and Kalman-based channel tracking is explored. The channel tracking is initialised, and interleaved every $K=\{12,24\}$ symbol periods a transmission of two pilot symbols and an MMSE channel estimate to re-initialised the Kalman tracking algorithm, incurring $8 \%$, and $4 \%$ loss in bandwidth eff ciency, respectively. Below, we compare the results for the simplif ed AR-2 model with the standard AR-1 Kalman f lter. Simulations are performed over an ensemble of $10^{4}$ randomised channel realisations with a maximum Doppler spread $\Omega_{D}=\{0.003 \pi, 0.005 \pi, 0.01 \pi\}$.

BER curves for a transmission system with a maximum Doppler spread $\Omega_{D}=0.003 \pi$ are shown in Fig. 3. For different update periods $K$, the AR-2 Kalman flter with solid BER curves has a small advantage over the AR-1 Kalman 


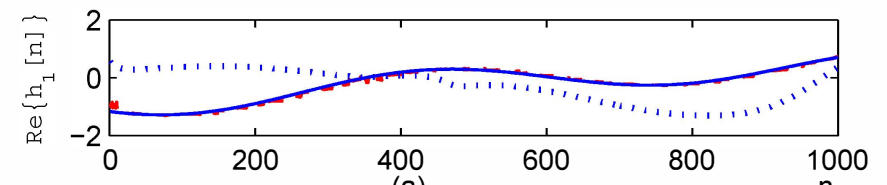

(a)

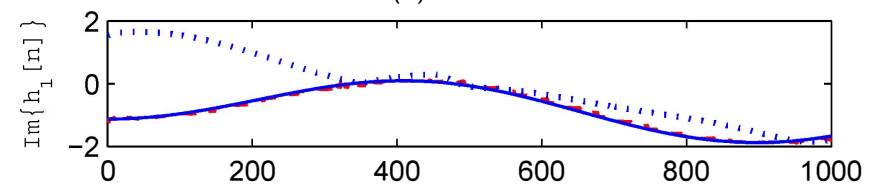

(b)
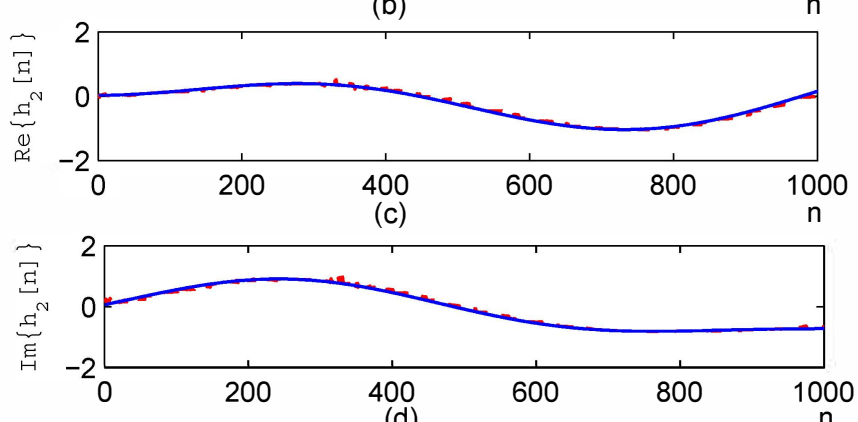

(d)

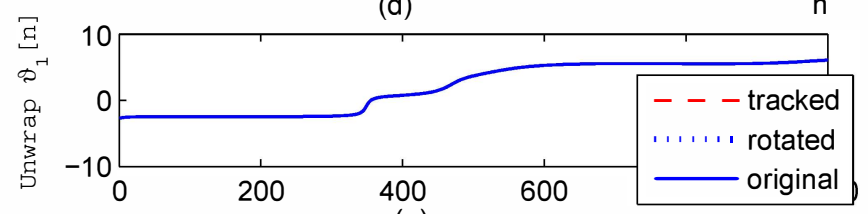

(e)

Figure 2. Coeff cient evolution of channel coeff cients - channel one with (a) real and (b) imaginary part, channel two with (c) real and (d) imaginary part, and (e) evolution of the angle returned to the transmitter via feedback.

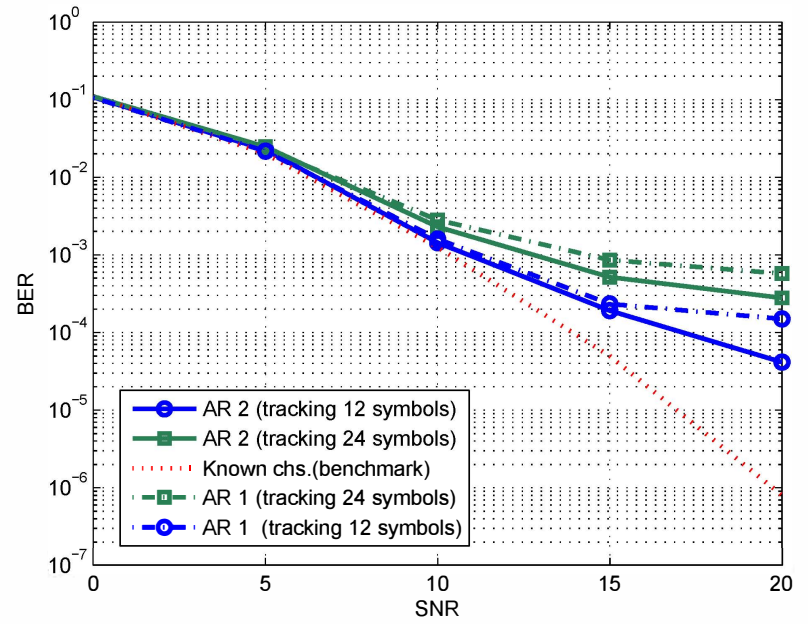

Figure 3. BER performance of EO-STBC system with channel estimation and tracking based on an AR-1 (dashed) and AR-2 (solid) Kalman flter for channel variations with $\Omega_{D}=0.003 \pi$.

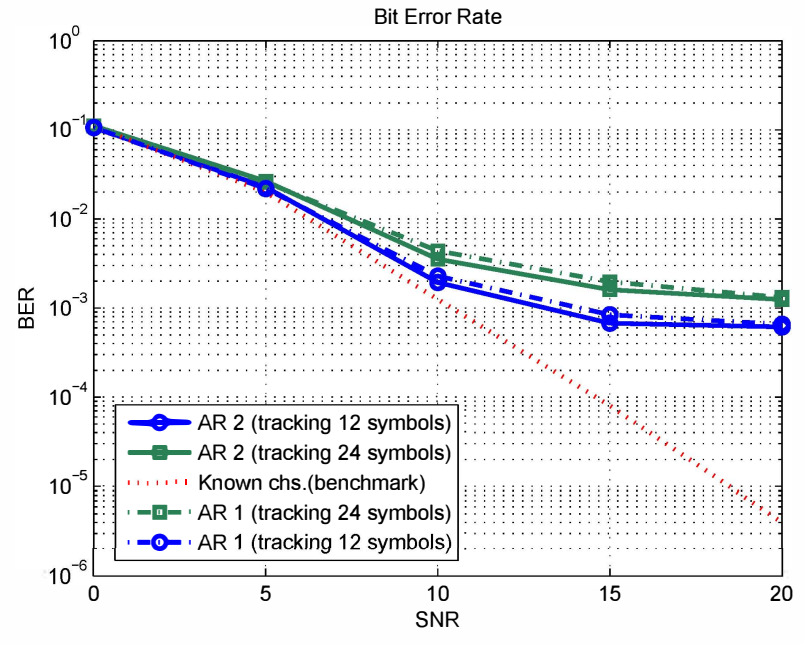

Figure 4. BER performance of EO-STBC system with channel estimation and tracking based on an AR-1 (dashed) and AR-2 (solid) Kalman flter for channel variations with $\Omega_{D}=0.005 \pi$.

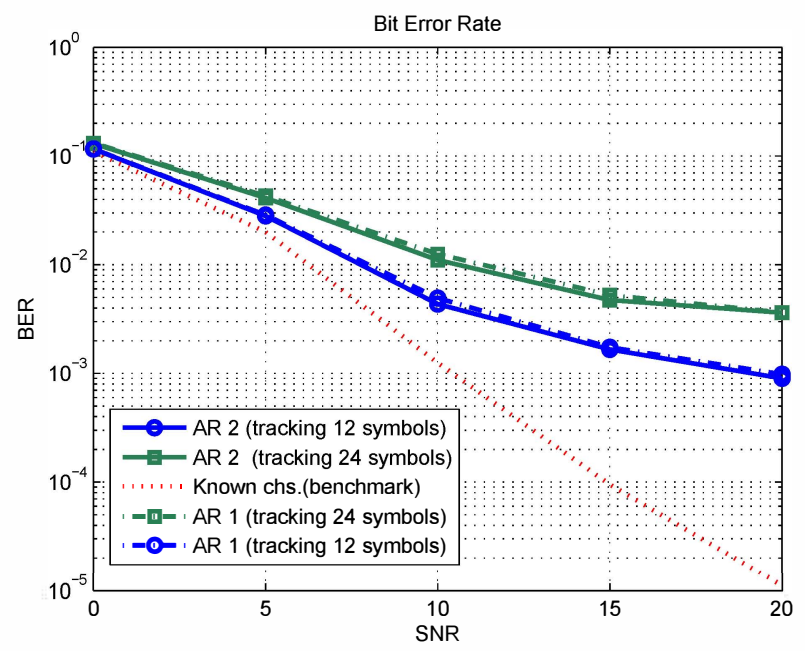

Figure 5. BER performance of EO-STBC system with channel estimation and tracking based on an AR-1 (dashed) and AR-2 (solid) Kalman flter for channel variations with $\Omega_{D}=0.01 \pi$.

approach. Particularly at high SNR, a signif cant advantage for the AR-2 system can be noted. The dashed line belongs to a genie aided system with perfect knowledge of CSI at both transmitter and receiver.

Fig. 4 shows the same BER performance for a higher maximum Doppler spread $\Omega_{D}=0.005 \pi$. The performance of the AR-2 Kalman f lter (solid curves) still outperforms the AR-1 model (dashed curves), but the higher rate of channel variation has reduced the benef $t$ compared the results shown in Fig. 3 for the lower variation.

Finally, a maximum Doppler spread of $\Omega_{D}=0.01 \pi$ leads to the performance characterised in Fig. 5. The performances of both AR-1 and AR-2 are very similar, with a negligible advantage for the AR-2 model, which provide little benef $t$ in a faster time-varying channel. 


\section{CONCLUSIONS}

In this paper, we have considered EO-STBC with beamsteering in order to maximise the joint diversity and array gain of such a MIMO transceiver. EO-STBC requires feedback of the steering angles, which can be calculated from channel estimates at the receiver. Since the channel requires to be estimated in order to decode the EO-STBC signals with maximum benef $t$, we have discussed a Kalman estimator to track the channel coeff cients. Their smooth variation has previously motivated a differentially encoded feedback of steering angles [8], and here additionally set the incentive to replace the AR-1 state-space model of the basic Kalman channel estimator with an AR-2 model that is capable of imposing additional smoothness.

Simulation results have shown that the overall EO-STBC system achieves suitable BER values, and that the performance of the proposed system offers a distinct advantage for lower Doppler spreads and the inclusion of an AR-2 model instead of an AR-1 model.

For the fnal paper, we are aiming to generate more detailed results, and analyse the system w.r.t. achievable BER performances for the case of errors only due to the temporal variation of the system.

\section{REFERENCES}

[1] S. M. Alamouti, "A Simple Transmit Diversity Technique for Wireless Communications," IEEE Journal on Selected Areas in Communications, vol. 16, pp. 1451-1458, Oct. 1998

[2] V. Tarokh, H. Jafarkhani, and A. Calderbank, "Space-time block codes from orthogonal designs," IEEE Transactions on Information Theory, vol. 45, pp. 1456-1467, July 1999.

[3] H. Jafarkhani, "A quasi-orthogonal space-time block code," IEEE Transactions on Communications, vol. 49, pp. 1-4, January 2001.

[4] C. Yuen, Y. L. Guan, and T. T. Tjhung, "Full-rate full-diversity STBC with constellation rotation," in 57th IEEE Semiannual Vehicular Technology Conference, Spring, vol. 1, (Jeju, Korea), pp. 296-300, April 2003.

[5] M. Celebi, S. Sahin, and U. Aygolu, "Full rate full diversity space-time block code selection for more than two transmit antennas," IEEE Transactions on Wireless Communications, vol. 6, pp. 16-19, January 2007.

[6] N. E. Eltayeb, Space-Time Coding for Broadband Point-toPoint and Collaborative Wireless Communications. PhD thesis, Loughborough University, January 2009.

[7] K. Baddour and N. Beaulieu, "Autoregressive modeling for fading channel simulation," IEEE Transactions on Wireless Communications, vol. 4, pp. 1650-1662, July 2005.

[8] M. N. Hussin and S. Weiss, "Extended orthogonal space-time block coded transmission with quantised differential feedback," in 7th International Symposium on Wireless Communication Systems, pp. 179-183, September 2010.

[9] C. Budianu and L. Tong, "Channel estimation for space-time orthogonal block codes," IEEE Transactions on Signal Processing, vol. 50, pp. 2515-2528, October 2002.

[10] B. Hughes, "Differential space-time modulation," IEEE Transactions on Information Theory, vol. 46, pp. 2567-2578, November 2000 .

[11] Z. Liu, G. B. Giannakis, and B. L. Hughes, "Double differential space-time block coding for time-selective fading channels," IEEE Transactions on Communications, vol. 49, pp. 1529-1539, September 2001.

[12] Z. Liu, X. Ma, and G. B. Giannakis, "Space-time coding and Kalman fltering for time-selective fading channels," IEEE Transactions on Communications, vol. 50, pp. 183-186, February 2002 .
[13] S. Haykin, K. Huber, and Z. Chen, "Bayesian sequential state estimation for MIMO wireless communications," Proceedings of the IEEE, vol. 92, pp. 439-454, March 2004.

[14] L. Ros, H. Hijazi, and E. P. Simon, "Paths complex gain tracking algorithms for OFDM receiver in slowly-varying channels," in 4th International Symposium on Communications, Control and Signal Processing, (Cyprus), pp. 1-6, March 2010.

[15] G. H. Golub and C. F. Van Loan, Matrix Computations. Baltimore, Maryland: John Hopkins University Press, 3rd ed., 1996. 